\title{
Inappropriately high left ventricular mass: marker of very high cardiovascular risk in patients with chronic kidney disease?
}

\author{
Emilio Nardi, Alessandro Palermo and Giuseppe Mulè \\ Hypertension Research (2012) 35, 800-801; doi:10.1038/hr.2012.64; published online 10 May 2012
}

$\mathrm{T}$ here is increasing knowledge of the burden of cardiovascular (CV) diseases affecting patients with chronic kidney disease (CKD). CV diseases are the main cause of death in the CKD population, and the majority of patients with CKD die before ever reaching the end-stage renal disease; in fact, for patients with CKD, the risk of a fatal $\mathrm{CV}$ event is much higher than the risk to develop end-stage renal disease. ${ }^{1,2}$ Although patients with CKD manifest a high prevalence of traditional CV risk factors, this does not fully account for the burden of $\mathrm{CV}$ diseases in CKD. Other factors, which are typical of the CKD condition, such as secondary hyperparathyroidism, anaemia or oxidative stress, are associated with enhanced CV damage and mortality. ${ }^{3}$ Traditional CV risk factors, however, have a central role. Hypertension, often together with diabetes, is today the first cause of CKD. Hypertension has a major role in cardiac damage in CKD via induction of the left ventricular hypertrophy $(\mathrm{LVH}),{ }^{4}$ whose prevalence is very high in patients with advanced CKD, with very high values of $L V$ mass ${ }^{5}$ (Figure 1 ). As in other populations, in CKD patients, the presence of $\mathrm{LVH}$ is predictive of a worse CV prognosis. $^{6}$

In recent years, the traditionally defined LVH has been joined by the concept of 'inappropriate' LV mass, resulting from the ratio of observed to predicted LV mass. From a haemodynamic view, LVH is primarily

Dr E Nardi and A Palermo and G Mulè are at the Dipartimento di Medicina Interna e Specialistica (DIMIS), Excellence Centre of the European Society of Hypertension, Università degli Studi di Palermo, Via Alcide De Gasperi 30-90146 Palermo, Italy

E-mail: emilionardi@virgilio.it considered an adaptive remodelling process, compensating for an increase in cardiac work. In this view, LV mass can be considered as inappropriate (i.e., inappropriately high) when exceeding the amount needed to adapt to stroke work for a given gender and body size; in detail, LV mass is defined as inappropriate when the ratio observed/predicted LV mass is $>128 \%{ }^{7}$

In this regard, it should be highlighted that LV growth, and eventually the development of LVH, depend not only on haemodynamic factors, but also on several non-haemodynamic factors (that remain in part unidentified), including genes and neuro-humoral systems. In this complex picture, some pathological conditions, such as $\mathrm{CKD}$, may have a very important role contributing to the structural changes of the left ventricle that lead to the development of LVH and/or of inappropriately high LV mass, and most importantly, in determining the functional LV alterations that may pave the way to overt heart failure.
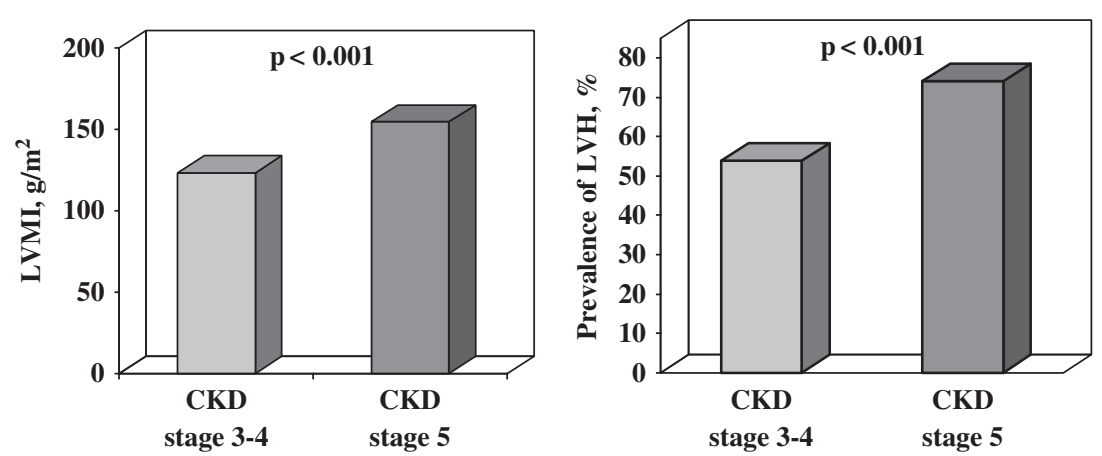

Figure 1 Left ventricular mass indexed by body surface area (LVMI) and prevalence of left ventricular hypertrophy (LVH) in patients with different stages of chronic kidney disease (unpublished personal data).

In this issue of Hypertension Research, the article by Chen et al. ${ }^{8}$ investigates the CV prognostic importance of the ratio observed/ predicted LV mass in 485 patients with CKD stage $3-5$. Previous papers by other research groups have shown that inappropriate LV mass has a negative impact on $\mathrm{CV}$ prognosis of hypertensive patients ${ }^{9}$ and is linked with the presence and severity of CKD. ${ }^{5,10}$ In the interesting study by Chen et al., ${ }^{8}$ inappropriate LVM is highly prevalent $(68.5 \%)$ and is independently related to CKD severity, in agreement with previous findings. ${ }^{5,10}$ Further, the presence of inappropriately high LV mass is independently associated with $\mathrm{CV}$ events. ${ }^{8}$ Other independent predictors of $\mathrm{CV}$ events in this study are old age, history of coronary artery disease, heart failure or atrial fibrillation, wide pulse pressure, low albumin, low haemoglobin and left atrium diameter. ${ }^{8}$ Without any doubt, this study gives interesting findings and expands existing data on the topics of the 
relationships between $\mathrm{LV}$ mass and $\mathrm{CKD}$, and of $\mathrm{CV}$ risk of patients with CKD.

However, the reading of papers focussing on inappropriate LV mass may sometimes be accompanied by silent questions in the reader's mind:

Is the evaluation of inappropriateness of LV mass indeed reliable?

In regard to this question, the predicted $\mathrm{LV}$ mass is estimated by means of an equation including height $(\mathrm{m}),{ }^{2,7}$ gender and stroke work. Stroke work is estimated starting from stroke volume and systolic blood pressure (BP)..$^{7}$ The reliability of this evaluation has been tested in a study by Muiesan et al. ${ }^{11}$; in this study, a difference of -22 or $+20 \%$ in the ratio of observed/predicted LV mass, measured on two different days, had a $90 \%$ probability to identify a true biological change. However, in our opinion, it is incontestable that a single BP measurement may have a great impact on the calculation of the predicted (i.e., 'appropriate') LV mass. In other words, should the single BP measurement obtained on the day of the echocardiographic examination be considered as representative of the BP load that, during months or years, contributed to cause the increase of LV mass in that patient? This can be considered the main limitation of the concept and use of inappropriate LV mass. In this regard, Chen et al. ${ }^{8}$ suggest that the use of average ambulatory BP instead of a single clinical BP measurement could be more reliable. Further, they correctly acknowledge that also low LV ejection fraction may influence the calculation of inappropriate LV mass.

Despite these limitations, inappropriate LV mass has shown to be associated with $\mathrm{CV}$ prognosis in different populations, ${ }^{8-9}$ thus it seems to correspond to something biologically plausible. In this regard, and adding a provocative comment, it should be noted that the concept of inappropriate LV mass could be in part comparable to the concept of very high LV mass, and the observation that CV risk increases linearly with the increase of LV mass is yet known in the literature. ${ }^{12}$ Beyond these concerns on the 'inappropriate' LV mass, it is still topical to draw attention on $\mathrm{CV}$ risk in patients with $\mathrm{CKD}$, and on the need for early and effective treatment in this population.

1 Hostetter TH. Chronic kidney disease predicts cardiovascular disease. N Engl J Med 2004; 351: 1344-1346.

2 Shulman NB, Ford CE, Hall WD, Blaufox MD, Simon D, Langford HG, Schneider KA. Prognostic value of serum creatinine and effect of treatment of hypertension on renal function: Results from the Hypertension Detection and Follow-up Program. The Hypertension Detection and Follow-up Program Cooperative Group. Hypertension 1989; 13(Suppl 5): S180-S193.

3 Raggi P, Boulay A, Chasan-Taber S, Amin N, Dillon M, Burke SK, Chertow GM. Cardiac calcification in adult hemodialysis patients. A link between end-stage renal disease and cardiovascular disease? J Am Coll Cardiol 2002; 39: 695-701.

4 Locatelli F, Bommer J, London GM, Martìn-Malo A, Wanner C, Yaqoob M, Zoccali C. Cardiovascular disease determinants in chronic renal failure: clinical approach and treatment. Nephrol Dial Transplant 2001; 16: 459-468.

5 Nardi E, Palermo A, Mulè G, Cusimano P, Cottone S, Cerasola G. Left ventricular hypertrophy and geometry in hypertensive patients with chronic kidney disease. J Hypertens 2009; 27: 633-641.

6 Foley RN, Parfrey PS, Harnett JD, Kent GM, Murray DC, Barre PE. The prognostic importance of left ventricular geometry in uremic cardiomyopathy. J Am Soc Nephrol 1995; 5: 2024-2031.

7 de Simone G, Devereux RB, Kimball TR, Mureddu GF, Roman MJ, Contaldo F, Daniels SR. Interaction between body size and cardiac workload: influence on left ventricular mass during body growth and adulthood. Hypertension 1998; 31: 1077-1082.

8 Chen S-C, Chang J-M, Liu W-C, Chen Y-Y, Chen L-I, Huang J-C, Yang T-K, Su H-M, Chen H-C. The ratio of observed to predicted left ventricular mass is independently associated with increased cardiovascular events in patients with chronic kidney disease. Hypertens Res 2012; 35: 832-838.

9 Muiesan ML, Salvetti M, Paini A, Monteduro C, Galbassini G, Bonzi B, Poisa P, Belotti E, Agabiti Rosei C, Rizzoni D, Castellano M, Agabiti Rosei E. Inappropriate left ventricular mass changes during treatment adversely affects cardiovascular prognosis in hypertensive patients. Hypertension 2007; 49: 1077-1083.

10 Cioffi G, Tarantini L, Frizzi R, Stefenelli C, Russo TE, Selmi A, Toller C, Furlanello F, de Simone G. Chronic kidney disease elicits excessive increase in left ventricular mass growth in patients at increased risk for cardiovacular events. J Hypertens 2011; 29: 565-573.

11 Muiesan ML, de Simone G, Ganau A, Longhini C, Verdecchia P, Mancia G, Agabiti-Rosei E. Working Group on Heart and Hypertension of Italian Society of Hypertension. Inappropriate LV mass: reliability and limitations of echocardiographic measurement for risk stratification and follow-up in single patients. J Hypertens 2006; 24: 2293-2299.

12 Schillaci G, Verdecchia P, Porcellati C, Cuccurullo O, Cosco C, Perticone F. Continuous relation between left ventricular mass and cardiovascular risk in essential hypertension. Hypertension 2000; 35: 580-586. 